\title{
DESAFIOS E RESULTADOS DURANTE IMPLEMENTAÇÃO DE FERRAMENTAS LEAN
}

\section{CHALLENGES AND RESULTS FROM A LEAN MANUFACTURING IMPLEMENTATION}

\author{
Alexandre Svenssno Gonçalves \\ Universidade Federal Tecnológica do Paraná - UTFPR - Curitiba - Brasil \\ goncalves_alexandre_s@cat.com
}

\begin{abstract}
Resumo
A crescente concorrência entre as empresas de manufatura advindas principalmente da abertura dos mercados e também da globalização, está acelerada pelo salto tecnológico nos meios de comunicação e também de automação, impulsionou a necessidade de melhorias de processos e eficiência nas empresas. Cada vez mais, é possível perceber que o mercado tem demandado produtos com qualidade superior, entregas mais rápidas e custo competitivo, sendo assim, melhorias de processos e eficiência são extremamente importante para assegurar a competitividade deste segmento. Diante disso a Manufatura Enxuta e conceitos sobre o Sistema Toyota de Produção tem sido um dos meios que as empresas têm utilizado para se tornarem mais eficientes, através de conceitos como: Zero Defeito, Eliminação de perdas, 5S, Manutenção Produtiva Total e Produção Puxada. Para entender como estes conceitos de Manufatura Enxuta e do Sistema Toyota de produção podem tornar uma empresa mais eficiente e competitiva, este trabalho apresentará um estudo de caso, que visa descrever dificuldades, desafios e resultados obtidos durante um processo de implementação de algumas ferramentas do Sistema Toyota de Produção.
\end{abstract}

Palavras-chave: Manufatura enxuta; Zero defeito; Melhoria Contínua; Produção Puxada; Sistema Toyota de Produção

\section{INTRODUÇÃO}

"A Máquina que Mudou o Mundo" consistiu em um importante livro responsável por disseminar os principais conceitos sobre manufatura enxuta e o Sistema Toyota de Produção (STP) (Womack, Jones, \& Ross, 2004), além de demonstrar como a empresa ficou conhecida a partir de suas práticas produtivas inovadoras. Segundo Shah e Ward (2003), a produção enxuta é uma maneira multidimensional que contempla uma vasta variedade de práticas de gerenciamento, das quais podemos destacar Just in Time, Sistema de Qualidade, Times de trabalho, células de manufatura, gerenciamento de fornecedores e outras ferramentas, geralmente conectadas em um sistema integrado. Saurin e Ferreira (2008) reforçam que a manufatura enxuta tem como objetivo a 
eliminação de qualquer perda do sistema de produção, possibilitando produtos e serviços de alta qualidade, ao menor custo possível e atendendo da melhor forma às necessidades dos clientes.

Neste contexto, considerando a necessidade de pesquisas e estudos que avaliem experiências durante a implementação de práticas de manufatura enxuta, especialmente no contexto brasileiro, este trabalho tem como objetivo principal a avaliação das dificuldades, desafios e resultados durante a implantação de conceitos Lean em uma empresa fabricante de máquinas de construção. Pode-se destacar que a empresa investigada iniciou esta jornada de implementação em 2002, quando decidiu desenvolver seu próprio sistema de manufatura, baseado nas práticas do STP. Além disso, o estudo de caso também pretende contribuir para o desenvolvimento do conceito de manufatura enxuta em pequenas e médias empresas, já que os exemplos neste segmento ainda são incipientes.

Este trabalho reveste-se de importância ao explorar e analisar experiências vivenciadas durante o processo de implementação das ferramentas Lean. Além disso, o compartilhamento de tais informações, por meio das discussões aqui realizadas, poderá servir de base para que outras empresas tomem conhecimento sobre as especificidades relativas ao tema aqui desenvolvido. Em termos de estrutura este trabalho se encontra assim apresentado além desta introdução: um capítulo sobre os principais conceitos e referenciais sobre a filosofia lean; posteriormente são apresentados os procedimentos metodológicos de pesquisa; na sequência se observam a apresentação e análise dos dados; e, por fim, são traçadas algumas considerações finais na conclusão do artigo.

\section{FILOSOFIA LEAN}

A Toyota com seu Sistema Toyota de Produção (STP) iniciou o novo conceito de sistema de produção enxuta, que de acordo seu fundador, Ohno (1997), tem como alicerce a absoluta eliminação do desperdício.

Ohno (1997) e Hines e Taylor (2000) destacam a existência de sete tipos de desperdícios básicos na operação, são eles: superprodução, fila, transporte ou movimentações desnecessárias, excesso de processamento ou processamento incorreto, excesso de estoque, e defeitos, que devem ser eliminados para aumentar a eficiência de operação. Para se alcançar a eliminação total desses desperdícios através da Produção Enxuta, é preciso uma boa implantação dos dois pilares de sustentação desse sistema, que são a Automação (principio pelo qual uma máquina automática é capaz de interromper seu processo sempre que ocorrer qualquer anomalia) e o Just-In-Time (meio através do qual as peças necessárias só chegam a seu ponto de uso apenas na quantidade necessária e no tempo necessário, possibilitando trabalhar com um inventário bem próximo de zero) (OHNO, 1988). 
Por muito tempo o tal sistema foi confundido com o sistema Kanban que é um meio para se chegar ao Just in time (JIT), um dos pilares do STP, caracterizado ou mesmo apresentado muitas vezes a partir da nomenclatura de Produção Enxuta ou lean Manufacturing. Womack e Jones (1998) definem a produção enxuta como um processo de cinco passos: definir o valor do cliente, definir o fluxo de valor, fazê-lo "Fluir", a "Puxar" a partir do cliente e lutar pela excelência.

Para Ohno (1988), os valores sociais mudaram, visto que o produto só será vendido se realmente agregar algum tipo de valor aos consumidores, já que cada cliente tem conceitos e gostos diferentes. Em consequência disso, o mundo industrial foi forçado a dominar significativamente o sistema de produção múltiplo, em pequenas quantidades.

\subsection{FERRAMENTAS DO SISTEMA TOYOTA DE PRODUÇÃO}

De acordo com Jabbour (2014) existem várias práticas que levam ao sucesso do STP, as quais são: Melhoria contínua, Produção Puxada/Just in Time, Gerenciamento da Qualidade via conceito de Zero defeito, 5S, Mapeamento do Fluxo de Valor e outros. Em função das ferramentas adotadas no estudo de caso investigado neste trabalho vamos destacar apenas os conceitos de Produção Puxada, Qualidade Zero Defeito e Mapeamento do Fluxo de Valor (MFV).

\subsubsection{PRODUÇÃO PUXADA E JUST IN TIME}

Para Monden (1984) Just-in-Time significa produzir as unidades necessárias em quantidades necessárias no tempo necessário. O referido autor ainda afirma que o principal propósito do sistema é reduzir os custos, mas também ajuda a aumentar o giro de capital e melhora a produtividade total da companhia. Segundo Correa (1993), o principal objetivo do sistema JIT é a melhoria contínua. Os estoques têm sido usados para evitar descontinuidades no processo produtivo, as quais são causadas por três tipos de problema: de qualidade, de quebra de máquina e de preparação de máquina. Reduzindo os estoques intermediários, estes problemas tornam-se cada vez mais visíveis, e desta forma, o JIT contribui para a identificação dos problemas, tornando mais fácil eliminá-los.

\subsubsection{MELHORIA DA QUALIDADE - CONCEITO DE ZERO DEFEITO}

Guinato (1996) descreve que o conceito de qualidade Zero defeito utilizado no STP é um método racional e científico capaz de eliminar a recorrência de defeitos através da identificação e controle das causas. Para Shingo (1986), são quatro os pontos importantes para a manutenção da filosofia de Zero Defeito, conforme demonstra o quadro abaixo. 
Quadro 1 - Pontos para a manutenção da filosofia de Zero Defeito.

1. Utilização da inspeção na fonte. Este método de inspeção tem caráter preventivo, capaz de eliminar completamente a ocorrência de defeitos, pois a função controle é aplicada na origem e não sobre os resultados.

2. Utilização de inspeção $100 \%$ ao invés de inspeção por amostragem.

3. Redução do tempo decorrido entre a detecção do erro e a aplicação da ação corretiva.

4. Reconhecimento de que os trabalhadores não são infalíveis. Aplicação de dispositivos à prova-defalhas ("Poka-Yoke") cumprindo a função de controle junto com a execução.

Fonte: SHINGO, Shigeo. Zero quality control: Source inspection and the poka-yoke system. Productivity Press, Cambridge, MA, USA, 1986.

A filosofia de Zero defeito determina que o sistema seja capaz de produzir continuamente produtos livres de defeitos. Este conceito, na Toyota, é aplicado a todas as operações e processos de forma que cada operação e cada processo sejam planejados considerando todas as possibilidades de defeito ou falha. Esta visão preventiva evita a geração de defeitos ao longo do processo produtivo.

\subsubsection{MAPEAMENTO DO FLUXO DE VALOR}

O Mapeamento do Fluxo de Valor (MFV) é uma ferramenta muito importante, e tem sido muito utilizada durante aplicações de ferramentas Lean. Este fluxo apresenta detalhadamente o conjunto de todas as atividades realizadas desde a compra de matéria prima até a entrega do produto final ao consumidor. Esta ferramenta, introduzida por Rother e Shook (1998), é um método de modelagem de empresas relativamente simples, com um procedimento para construção de cenários de manufatura. Esta modelagem leva em consideração tanto o fluxo de materiais como o fluxo de informações e ajuda bastante no processo de visualização da situação atual e na construção da situação futura. Em suma, o MFV é uma ferramenta que, assim como as outras da Produção Enxuta, se concentra mais nas questões relativas à redução do lead time dos sistemas.

Segundo Kaplan e Cooper (1998), é preciso uma análise racional que justifique também as vantagens e desvantagens econômicas derivadas do fato de se destinarem certos recursos para a melhoria de determinado processo de uma organização. Através do MFV você pode enxergar o fluxo, e com isso identificar mais do que os desperdícios. Mapear as operações ajuda a identificar as fontes do desperdício; fornece uma linguagem comum para tratar dos processos de manufatura; torna as decisões sobre o fluxo visíveis, de modo que você possa discuti-las; junta conceitos e técnicas enxutas, que ajudam a evitar a implementação de algumas técnicas isoladamente; forma a base para um plano de implementação; e mostra a relação entre o fluxo de informação e o fluxo de material.

A meta que se pretende alcançar pela Análise do Fluxo de Valor é a obtenção de um fluxo contínuo, orientado pelas necessidades dos clientes, e livre de desperdícios desde a matéria prima até o produto final. 


\subsection{DESAFIOS E IMPACTOS DURANTE A IMPLEMENTAÇÃO}

Abernathy et al. (2000), declara que o sucesso das empresas automotivas Japonesas, tal como a Toyota, pode ser atribuído ao cuidado no gerenciamento das pessoas, materiais e equipamentos. Já Liker (2004), destaca que a perseverança, planejamento de longo prazo e também a consciência de sacrificar benefícios de curto-prazo em benefício de maiores resultados no longo prazo são fundamentos importantes do STP, e que contribuíram demasiadamente para o sucesso das implementações. Para Bhasin e Burcher (2006), as ferramentas Lean devem ser introduzidas de maneira simultânea, pois reforçam o ponto crítico de entender a filosofia da manufatura enxuta como uma jornada sem fim e não como um estado final que a organização tentará alcançar.

O sistema Just in Time tem uma parcela importante durante a implementação do Lean, entretanto não se pode olhar da cerca da empresa para dentro somente, mas sim para toda a cadeia de abastecimento. Sendo assim, isso dependerá da participação de clientes e fornecedores e de toda a infraestrutura que liga estes elementos. Lewis (2000), diz que cada empresa terá sua própria trajetória no desenvolvimento do Lean, e consequente seu próprio sistema Lean de produção. Além disso, o modelo a ser implementado não precisa ser tudo ou nada, mas sim customizado para o modelo de negócio da empresa disposta a iniciar este conceito.

Segundo Rymaszewska (2014), o sucesso durante a adoção vai requerer planejamento e acompanhamento de longo prazo, em troca de resultados sustentáveis, mas que demoram mais para se concretizar, entretanto fazem parte do processo de criação de cultura. $\mathrm{O}$ desenvolvimento da cultura de manufatura enxuta normalmente é o grande desafio durante as implementações, uma vez que novos conhecimentos precisam ser criados dentro da organização, para expansão e sedimentação dos conceitos.

\section{PROCEDIMENTOS METODOLÓGICOS}

Pesquisa é o caminho para se chegar à ciência e ao conhecimento. É na pesquisa que utilizaremos diferentes instrumentos para se chegar a uma resposta mais precisa. Conforme Ribeiro (2008), o instrumento que o pesquisador utilizará para atingir resultados ideais será estipulado por ele mesmo.

A partir de uma abordagem qualitativa, este trabalho utilizará o estudo de caso para descrever as dificuldades, desafios e resultados obtidos durante um processo de implementação de ferramentas Lean. O estudo de caso é um estudo de natureza empírica que investiga um determinado fenômeno, geralmente dentro de um contexto real do cotidiano. Trata-se de uma análise aprofundada de um ou mais objetos (casos), para que permita o seu amplo e detalhado conhecimento (GIL, 1996; BERTO; NAKANO, 2000). Seu objetivo é aprofundar o conhecimento 
acerca de um problema não suficientemente definido (MATTAR, 1996), visando estimular a compreensão, sugerir hipóteses e questões ou desenvolver a teoria.

Em geral, os estudos de casos podem ser classificados segundo, Yin (2001) e Voss et al. (2002): pelo conteúdo e objetivo final (exploratórios, explanatórios, ou descritivos) ou quantidade de casos (caso único - holístico ou incorporado). A principal tendência em todos os tipos de estudo de caso, é que estes tentam esclarecer o motivo pelo qual uma decisão ou um conjunto de decisões foram tomadas, como foram implementadas e com quais resultados alcançados (YIN, 2001). Após os contatos, os dados devem ser coletados utilizando os instrumentos definidos no planejamento. As habilidades de entrevistas devem ser consideradas, a partir dos seguintes fatores (YIN, 2001): ter capacidade de fazer questões adequadas e interpretar as respostas; ser um bom ouvinte e não trazer nenhum tipo de preconceito; estar muito bem embasado (teoricamente) no tema sendo investigado; ser receptivo e sensível a possíveis evidências contraditórias; ser adaptável e flexível às situações novas e não previstas, considerando-as como oportunidades e não ameaças.

O método utilizado neste trabalho foi de pesquisa empírica, com dados coletados em campo. O Objetivo foi expandir o conhecimento sobre a aplicação empírica do conceito de manufatura enxuta, e princípios do STP a fim de entender os desafios e resultados durante as fases de implementação. Para Rosa e Arnoldi (2006) a pesquisa é uma atividade de investigação capaz de oferecer e produzir um conhecimento novo a respeito de uma área ou de um fenômeno, sistematizando-o em relação ao que já se sabe.

A coleta de dados foi realizada por meio de pesquisa documental e entrevistas semiestruturadas. A pesquisa documental teve o objetivo de buscar o nível de desempenho de alguns processos antes da implementação e compará-los com os resultados pós-implementação. Os documentos utilizados foram relatórios de desempenho, tais como: Defeitos por unidade produzidos, medições de lead time de produção antes e depois, quantidade de materiais comprados via JIT e dados sobre giros de inventário. Segundo Godoy (1995), na pesquisa documental, três aspectos merecem atenção especial por parte do investigador: a escolha dos documentos, o acesso a eles e a sua análise. Já as entrevistas foram conduzidas com a liderança da empresa pesquisada e também no chão de fábrica com os empregados participantes do processo de implementação. Para Gil (1999), a entrevista é seguramente a mais flexível de todas as técnicas de coleta de dados de que dispõem as ciências sociais. Foram realizadas nove entrevistas, sendo elas distribuídas da seguinte maneira: 4 funcionários da produção, 2 da qualidade e 3 gerentes. As entrevistas foram realizadas na fábrica, durante a visita as células de trabalho e duraram em média 40 minutos cada uma. $\mathrm{O}$ conteúdo das entrevistas foi transcrito para posterior análise e utilização na apresentação dos resultados. 


\section{APRESENTAÇÃO E ANÁLISE DOS DADOS}

\subsection{DESCRIÇÃO HISTÓRICA DA EMPRESA ESTUDADA}

A empresa estudada está localizada na região metropolitana de Curitiba, e atua no ramo de fabricação de máquinas e equipamentos de terraplenagem. Atualmente conta com uma estrutura fabril moderna, organizada e flexível, que proporciona tecnologia, qualidade e alta velocidade para atender às necessidades dos clientes. A empresa adota os mais modernos conceitos de excelência para flexibilizar suas operações, produzir cada vez melhor e oferecer produtos e serviços da mais alta qualidade.

\subsubsection{O Conceito de Manufatura Enxuta na empresa}

O Sistema de Produção da empresa estudada foi customizado, utilizando como base os conceitos do STP. Este sistema de produção visa garantir um processo eficiente e livre de perdas desde o momento em que o cliente faz o pedido até a entrega do produto final. Existem três pilares fundamentais para a sustentação do sistema, que são: Sistema Operacional, Cultural e Gerencial, que funcionando conjuntamente focam na identificação e eliminação das oito perdas, garantindo que a empresa seja reconhecida por sua excelência em segurança, qualidade e velocidade.

\subsection{IMPLEMENTAÇÃO - PONTOS RELEVANTES}

\subsubsection{MUDANÇA CULTURAL}

A mudança cultural durante um processo de aplicação de conceitos de manufatura enxuta é muito importante e precisa contar com o comprometimento da liderança para que o programa tenha sucesso. Este item foi mencionado pelos líderes durante as entrevistas, os quais mencionaram que o progresso da implementação e sustentabilidade da disciplina depende fundamentalmente do comprometimento de todos os níveis de liderança, os quais irão manter a cultura anos após anos, criando um ambiente de aprendizado contínuo.

Adicionalmente ao papel da liderança, foi identificada a importância de um programa robusto de treinamento e capacitação dos empregados nos conceitos de Manufatura enxuta. O programa de treinamento e desenvolvimento precisa ser contínuo e vivo, pois precisa ser atualizado constantemente para garantir que os funcionários estarão sempre atualizados sobre os procedimentos que precisam seguir para boa realização de suas atividades. Foi encontrada nas células de trabalho uma matriz de habilidades, que gerencia o conhecimento e habilidades de cada operador em cada posto da célula de trabalho. A flexibilidade é fundamental para garantir um fluxo 
constante nos sistemas de produção, sendo assim o gerenciamento da mão de obra com relação às habilidades se torna necessário e fundamental.

\subsubsection{PRODUÇÃO PUXADA}

Foi verificado durante a visita á fabrica a presença de várias ferramentas que auxiliam na produção puxada, tais como:

- $\quad$ One Piece Flow - (fluxo puxado um a um) - Algumas células de trabalho possuem o layout que somente permite a movimentação de uma peça (uma unidade) entre as etapas de trabalho, sendo assim não existe espaço para superprodução. Existem marcações no chão, para controlar o WIP (Work in Process).

- $\quad$ Kitting - As linhas de montagem são abastecidas em aproximadamente $70 \%$ via sistema de Carros de Kit, com sistema de duas caixas, onde toda vez que uma fica vazia, inicia-se o processo de reabastecimento. O Kit é composto de várias peças diferentes que foram disponibilizadas de forma a facilitar a vida do operador que está na linha de montagem, e então extrair o máximo de eficiência na célula durante as atividades que realmente agregam valor ao produto.

- $\quad$ Esteiras - Foi verificada a presença de esteiras transportadoras em diversas áreas, que por gravidade movimentam peças de um ponto a outro, eliminando ou diminuindo a necessidade de movimentação pelo homem e reduzindo atividades que não agregam valor, neste caso transporte.

- Carrossel - Também foram encontrados vários carrosséis, onde carros transportadores são utilizados e empurrados pelos funcionários para movimentação de conjuntos pesados, evitando o uso de pontes rolantes, que na maioria das vezes adiciona perdas no processo produtivo além de aumentar o risco de acidentes.

- Linha de montagem automática - As linhas de montagem são movimentadas automaticamente, de acordo com seu respectivo Takt Time, e garantem ritmo ao fluxo de produção, este essencial para manter um fluxo constante e balanceado.

- $\quad$ Sistema JIT de compra - Encontramos diversos itens comprados via Just in Time, os quais garantem um estoque mínimo para atendimento da demanda.

As ferramentas acima descritas foram muito importantes para o progresso e desenvolvimento dos conceitos de manufatura enxuta na empresa estudada, pois algumas das ferramentas verificadas controlam automaticamente a manutenção do ambiente livre de desperdícios. Neste caso podemos destacar o sistema One Piece Flow, pois não permite excesso de 
material em processo, e a linha de montagem automática, pois assegura a continuidade e compasso do fluxo produtivo. Este estudo comprovou que as ferramentas são de aplicação simples, entretanto trazem resultados significativos, além de poderem ser aplicadas em vários segmentos da indústria, bem como em empresas de pequeno e médio porte, onde conceitos Lean ainda são praticamente inexistentes.

\subsubsection{MELHORIA DA QUALIDADE}

O conceito de Zero Defeito está presente no sistema da Qualidade da empresa estudada, e os pilares são os cinco apresentados a seguir:

1. Parar os defeitos na origem: Os próprios trabalhadores têm a função e responsabilidade de não aceitar defeitos, não produzir defeitos e não passar defeitos para a próxima estação. Os defeitos são em sua maioria resolvidos assim que são identificados. Durante a visita na linha de montagem, foi constatada a presença de dois portões de qualidade ao longo do processo, onde inspeções são realizadas ao longo do processo e previnem a possibilidade de defeitos somente serem encontrados no final do processo de produção, o que torna a solução ou reparo mais demorada e mais cara.

2. Descrição do Problema: Ter uma excelente descrição do problema, para facilitar o processo de análise de causa raiz. Nesta etapa é muito importante identificar e registrar as possíveis causas e não sintomas.

3. Identificar a área responsável pelo defeito: O Passo anterior é muito importante para esta fase, pois neste momento o problema é direcionado para a área causadora, a qual terá responsabilidade de se aprofundar na causa raiz e encontrar uma solução definitiva para a solução do problema. Uma ação de contenção precisa estar implementada em 24 horas e uma solução permanente precisa estar concluída em 30 dias.

4. Feedback imediato do defeito: Nesta etapa o ponto principal é comunicar o defeito assim que o mesmo for identificado no processo produtivo. Neste momento já existe uma tentativa de conter os defeitos na origem imediatamente. Durante a visita no local de trabalho identificamos um defeito, que foi causado por mão de obra, e neste caso o funcionário e toda a célula de trabalho foram comunicados imediatamente, para garantir que os defeitos seriam contidos daquele momento em diante.

5. Solução do Problema: Nesta etapa é necessária a realização de uma análise de causa raiz, utilizando ferramentas como 5 porquês e $8 \mathrm{D}$, pois a área causadora tem prazos estabelecidos para implementar uma ação robusta e não mais deixar que aquele defeito aconteça. Para os problemas de gravidade média e alta, a área causadora precisa compartilhar com os executivos da empresa o resultado da análise e medidas tomadas para resolver o problema. 
Os fundamentos da qualidade verificados realmente tem papel fundamental para a manutenção da cultura de Zero Defeito. Os operadores são envolvidos em todas as etapas do processo, criando um senso de responsabilidade por aquilo que produzem e pela obsessão de produzir um produto livre de defeitos.

\subsubsection{FERRAMENTAS LEAN}

Inúmeras ferramentas oriundas do sistema Toyota de Produção, foram evidenciadas no processo produtivo investigado, tais como:

- $\quad 5 \mathrm{~S}-\mathrm{O}$ Conceito $5 \mathrm{~S}$ está presente em todas as áreas e foi verificado um bom nível de disciplina para manutenção dos outros S's. Esta ferramenta tem ajudado a tornar a vida dos funcionários mais fácil, pois sabem exatamente onde tudo está localizado e encontra-se em perfeito estado de funcionamento, contribuindo para a Qualidade e segurança das células de trabalho.

- $\quad$ Andon (ferramenta de Gerenciamento Visual) - Existem Andon's automatizados e manuais espalhados pela fábrica. Esta ferramenta ajuda muito no gerenciamento visual e tempo de resposta na solução de problemas. Em cada célula das linhas de montagem existem botões ligados ao sistema central, e quando um problema como disponibilidade de material, qualidade e outros é verificado, o funcionário aciona o botão, que imediatamente gera um sinal visual no quadro e também um sinal sonoro para chamar atenção do gestor da área, do operador de logística ou do analista de qualidade, os quais precisam atender ao local chamado para resolver a situação e reiniciar a produção.

- Heijunka - O processo de Vendas e Planejamento de produção verificado tem como premissa sempre buscar o nivelamento da produção, com o objetivo de trazer estabilidade para o processo de manufatura. O plano de produção é planejado para os próximos 14 meses, e este plano é congelado sempre pelos três primeiros meses. O sequenciamento de linha é congelado 20 dias antes das máquinas entrarem na linha de montagem.

- $\quad$ Mapeamento do Fluxo de Valor - Todas as células de trabalho têm seu fluxo de valor mapeado. A revisão do MFV é extremamente necessária, pois os projetos de melhoria contínua normalmente se iniciam durante o processo de revisão, o qual deve ocorrer anualmente ou quando uma oportunidade for identificada.

\subsection{RESULTADOS}


Por meio dos documentos acessados foi possível observar que a implementação das Cinco etapas para o Zero Defeito está trazendo resultados positivos na redução de defeitos gerados e/ou encontrados durante o processo produtivo.

Além da redução dos defeitos por unidade produzida, foi verificada uma redução de $40 \%$ no tempo de reparo por máquina. A combinação de redução de defeitos, redução no tempo de reparo e ganho de eficiência em todo o processo possibilitou uma redução de $20 \%$ no lead time de produção da fábrica nos últimos 12 meses.

A implementação do Just In time, trouxe muitos ganhos de eficiência no processo produtivo, pois apenas se mantem o necessário e respectivamente se movimenta somente o necessário. Melhorias no fluxo de materiais adicionadas ao Just in Time trouxeram melhorias de aproximadamente $15 \%$ na produtividade da mão de obra do setor de Logística, e as reduções de inventário chegaram a mais de $20 \%$ somadas as reduções de lead time mencionadas acima.

$\mathrm{Na}$ opinião dos entrevistados a melhoria contínua depende de uma mão de obra melhor qualificada, desta forma o desenvolvimento do time de trabalho precisa ser contínuo, para tornar o profissional preparado para assumir múltiplas funções quando necessário, mas de maneira planejada e organizada. A disciplina foi destacada como um desafio constante e que requer persistência, sendo assim os líderes tem papel importante em garantir a disciplina na execução dos processos, pois somente assim irão perpetuar os ganhos de eficiência e qualidade.

Em resumo, a integração da produção puxada, conceito de Zero Defeito, MFV, associadas às ferramentas Lean descritas acima formaram um conjunto harmonioso e eficiente, com resultados evidentes e efetivos, que podem contribuir para a melhoria de competitividade de qualquer empresa.

\section{CONCLUSÃO}

As técnicas de manufatura enxuta oriundas do Sistema Toyota de Produção, em geral trazem benefícios para as empresas que decidem verdadeiramente embarcar nesta jornada. Não se trata de uma receita simples, mas sim muito complexa, e que exige esforço, comprometimento da liderança e gestão de mudanças. Disciplina é um conceito chave para a manutenção da redução dos desperdícios e dos ganhos. O desenvolvimento e capacitação dos funcionários é peça chave para que a engrenagem gire de forma estável e consistente. Organizações que buscam competitividade, melhoria de eficiência e precisam melhorar a satisfação de seus clientes, seja para melhorar a qualidade, reduzirem o tempo de entrega ou entregar produtos com maior valor agregado certamente podem utilizar os conceitos da manufatura enxuta para alcançar seus objetivos. O tema 
Manufatura enxuta não se aplica somente a grandes organizações, sendo assim pequenas e médias empresas podem e devem desenvolver este conhecimento e consequentemente melhorar a eficiência de seus processos.

Abstract: The increasing competition among manufacturing companies arising mainly from the opening markets and globalization, which was accelerated by technological leap in the communication channels and also automation, spurred the need for processes and efficiency improvements in these companies. Increasingly, it's easy to realize that the market is demanding products with superior quality, faster delivery and competitive price, therefore, process improvements and efficiency are extremely important to ensure the competitiveness of this segment. Thus Lean Manufacturing and concepts of the Toyota Production System has been one of the ways that companies have used to become more efficient, through concepts such as: Zero Defect, waste reduction, 5S, Total Productive Maintenance and Pull Production. To understand how these concepts of Lean Manufacturing and the Toyota Production System can create a more efficient and competitive company, this paper presents a case study that aims to describe difficulties, challenges and results obtained during an implementation process that used some of the Toyota Production System tools.

Key-words: Lean Manufacturing; Zero defect; Continuous Improvement; Pull Production; Toyota Production System

\section{Referências}

ABERNATHY, Frederick H. et al. A stitch in time: Lean retailing and the transformation of manufacturing-lessons from the apparel and textile industries. Oxford University Press, 1999.

BERTO, Rosa Maria Villares S.; NAKANO, Davi Noboru. Revisitando a produção científica nos anais do Encontro Nacional de Engenharia de Produção. Production Journal, v. 24, n. 1, p. 225-232, 2014.

BHASIN, Sanjay; BURCHER, Peter. Lean viewed as a philosophy. Journal of manufacturing technology management, v. 17, n. 1, p. 56-72, 2006. crossref

DOROTA RYMASZEWSKA, Anna. The challenges of lean manufacturing implementation in SMEs. Benchmarking: An International Journal, v. 21, n. 6, p. 987-1002, 2014.

CORREA, Henrique Luiz; GIANESI, Irineu GN. Just in time, MRP II e OPT: um enfoque estratégico. Atlas, 1993.

GIL, Antonio Carlos. Métodos e técnicas de pesquisa social. In: Métodos e técnicas de pesquisa social. Atlas, 2010.

GHINATO, Paulo. Sistema Toyota de Produção: mais do que simplesmente just-in-time. Caxias do Sul: EDUCS, 1996.

GODOY, Arilda Schmidt et al. A pesquisa qualitativa e sua utilização em administração de empresas. Revista de Administração de Empresas, v. 35, n. 4, p. 65-71, 1995. 
HINES, Peter; TAYLOR, David. Going lean. Cardiff, UK: Lean Enterprise Research Centre Cardiff Business School, p. 3-43, 2000.

JABBOUR, Charbel José Chiappetta; SANTOS, Fernando César Almada. The central role of human resource management in the search for sustainable organizations. The International Journal of Human Resource Management, v. 19, n. 12, p. 2133-2154, 2008. crossref

KAPLAN, Robert S.; COOPER, Robin. Custo e desempenho: administre seus custos para ser mais competitivo. Futura, 1998.

LIKER, Jeffrey. K. O modelo Toyota: 14 princípios de gestão do maior fabricante do mundo. Porto Alegre, RS: Bookman, 2005.

LIKER, Jeffrey. K. The Toyota Way: 14 Management Principles from the World's Greatest Manufacturer, McGraw-Hill, New York, NY, 2004.

LEWIS, Michael A. Lean production and sustainable competitive advantage. International Journal of Operations \& Production Management, v. 20, n. 8, p. 959-978, 2000. crossref

MATTAR, Fauze Najib. Pesquisa de marketing: execução, análise. Atlas, 1998.

MONDEN, Yasuhiro. Produção sem estoques-Uma abordagem prática ao sistema de produção da Toyota. São Paulo: IMAM, 1984.

OHNO, Taiichi. O Sistema Toyota de Produção Além Da Produção. Bookman, 1997.

OHNO, Taiichi; MITO, Setsuo. Just in time for today and tomorrow. Productivity Press, 1988.

OSADA, Takashi. Housekeeping 5S's: seiri, seiton, seiso, seiketsu, shitsuke. 2. ed. IMAM . São Paulo, SP, 1995.

RIBEIRO, Haroldo. 5S housekeeping: um roteiro para uma implantação bem sucedida. 4. ed. Salvador, BA: Casa da Qualidade, 1994.

RIBEIRO, Elisa Antônia. A perspectiva da entrevista na investigação qualitativa. Revista Evidência, v. 4, n. 4, 2012.

ROSA, Maria Virgínia de F. P. do C.; ARNOLDI, Marlene Aparecida G. C. A entrevista na pesquisa qualitativa: mecanismos para a validação dos resultados. Belo Horizonte, MG, Autêntica Editora, p. 112, 2006.

ROTHER, Mike; SHOOK, John. Learning to see: value stream mapping to add value and eliminate muda. Lean Enterprise Institute, 2003.

SHAH, Rachna; WARD, Peter T. Lean manufacturing: context, practice bundles, and performance. Journal of operations management, v. 21, n. 2, p. 129-149, 2003., v. 21, n. 2, p. 129-149, 2003.

SAURIN, Tarcisio Abreu; FERREIRA, Cléber Fabrício. Avaliação qualitativa da implantação de práticas da produção enxuta: estudo de caso em uma fábrica de máquinas agrícolas. Gestão \& Produção, v. 15, n. 3, p. 449-462, 2008.

SANTOS, Luciano Costa; GOHR, Cláudia Fabiana; DOS SANTOS, Eder Jonis. Aplicação do mapeamento do fluxo de valor para a implantação da produção enxuta na fabricação de fios de cobre. Revista Gestão Industrial, v. 7, n. 4, 2012.

SHINGO, Shigeo. O Sistema Toyota de Producao Do Ponto. Bookman, 1996.

SHINGO, Shigeo. Zero quality control: Source inspection and the poka-yoke system. Productivity Press, Cambridge, MA, USA, 1986.

SIQUEIRA, Juliana. O sistema de custos como instrumento de apoio ao processo decisório: Um estudo multicaso em indústrias do setor metal-mecânico da Região Noroeste do Estado do Rio Grande do Sul. 2005. Tese de Doutorado. Dissertação de Mestrado apresentada à Universidade Regional do Noroeste do Estado do Rio Grande do Sul-UNIJUÍ, como requisito para obtenção do título de Mestre em Desenvolvimento, Gestão e Cidadania. Orientador: Dr. Ernani Ott. Ijuí. 
VOSS, Chris; TSIKRIKTSIS, Nikos; FROHLICH, Mark. Case research in operations management. International journal of operations \& production management, v. 22, n. 2, p. 195-219, 2002. crossref

JONES, Daniel T.; WOMACK, James P. A mentalidade enxuta nas empresas: elimine o desperdício e crie riqueza. Gulf Professional Publishing, 2004.

WOMACK, PJ JONES; JONES, Daniel T. DT ROSS D. A máquina que mudou o mundo, 1990.

YIN, Robert K. Estudo de Caso: Planejamento e Métodos. Bookman editora, 2015.

\section{Dados do Autor:}

Nome Completo: Alexandre Svenssno Gonçalves

Filiação institucional: Caterpillar Brasil Ltda

Cargo: Gerente da Cadeia de Abastecimento e Logística Interna

Endereço: Rua Monsenhor Ivo Zanlorenzi, 2520 - apto 901 - Curitiba - PR - CEP: 81.210.000

Telefones de contato: (41) 9612-2230 e (41) 3375-5620

e-mail: goncalves_alexandre_s@cat.com 